\title{
X-Linked Immunodeficiency with Increased IgM: Clinical, Ethnic, and Immunologic Heterogeneity
}

\author{
CATHERINE U. KYONG, G. VIRELLA, H. HUGH FUDENBERG, ${ }^{(31)}$ AND C. P. DARBY \\ Departments of Pediatrics (C.U.K., C.P.D.) and Basic and Clinical Immunology and Microbiology (G.V., H.H.F.), \\ Medical University of South Carolina, Charleston, South Carolina, USA
}

Summary

Two cases of immunodeficiency with increased IgM are reported. Patient 1 was a black male 3.5 years old who had recurrent pyogenic infections, failure to thrive, oral thrush, and systemic cryptococcal infection. Patient 2 was a 9-year-old white female who had recurrent cervical abscesses. Serum immunoglobulin determinations by radial immunodiffusion in both patients showed marked depression of IgG and IgA and marked elevation of IgM. A low molecular weight circulating monomeric IgM was demonstrated by immunoelectrophoresis and gel filtration in the second patient; this was not present in the first case. In vitro impairment of cellular immunity was observed in both patients. Administration of dialyzable leukocyte extracts (transfer factor) led to improvement of cell-mediated immunity in patient 1 . The etiology of this syndrome apparently has several different genetic bases. These patients demonstrate heterogeneity in genetic, ethnic, immunologic, and other features of the syndrome.

\section{Speculation}

The cause of hyper-IgM syndrome with depressed synthesis of IgA and IgG immunoglobulins is unknown. Deficiency in the function of $T$ cells ${ }^{1}$ or a subpopulation thereof could have a causal

${ }^{1}$ The abbreviations used are T cells: thymus-dependent lymphocytes; PHA: phytohemagglutinin; SKSD: streptokinase-strepto- relation to this syndrome, since there is increasing evidence that normal cellular immunity is necessary for the IgM-to-IgG "switch" in immunoglobulin production; whether it is also necessary for the formation of normal polymeric IgM from monomeric IgM is unknown, but this possibility merits speculation. In the present study, the occurrence of monomeric IgM in one patient but not the other, along with the differences in clinical manifestations, raises the possibility that the presence of monomeric IgM may be an index of a distinct subcategory of this syndrome with certain clinical features present only when the monomeric molecule is found. Our two patients, and all eight previously reported patients in whom isohemagglutinins were studied, had isohemagglutinins for $A$ and $B$ cells (i.e., were blood type $O$ ). In addition, two other hyper-IgM patients seen by us subsequently have been found to be blood type $O$ (unpublished observation). If this phenomenon is universal, it would suggest a very close association between the blood group $O$ antigen and this syndrome.

dornase; LIF: leukocyte migration inhibition factor; NBT: nitroblue tetrazolium dye test; LMAT: leukocyte migration inhibition test in agarose; ConA: concanavalin A; DLE: dialyzable leukocyte extract (dialyzable transfer factor); PPD: purified protein derivative of Mycobacterium bovis; LMW IgM: low molecular weight IgM; Cohn Fraction II: therapeutic $\gamma$-globulin. 